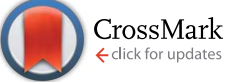

Cite this: Nanoscale, 2014, 6, 10140

\title{
Improved heterogeneous electron transfer kinetics of fluorinated graphene derivatives $\uparrow$
}

\begin{abstract}
Sidhureddy Boopathi, Tharangattu N. Narayanan* and Shanmugam Senthil Kumar*
Though graphitic carbons are commercially available for various electrochemical processes, their performance is limited in terms of various electrochemical activities. Recent experiments on layered carbon materials, such as graphene, demonstrated an augmented performance of these systems in all electrochemical activities due to their unique electronic properties, enhanced surface area, structure and chemical stabilities. Moreover, flexibility in controlling electronic, as well as electrochemical activities by heteroatom doping brings further leverage in their practical use. Here, we study the electron transfer kinetics of fluorinated graphene derivatives, known as fluorinated graphene oxide (FGO) and its reduced form, RFGO. Enhanced electron transfer kinetics (heterogeneous electron transfer (HET)) is observed from these fluorinated systems in comparison to their undoped systems such as graphene oxide (GO) and reduced GO. A detailed study has been conducted using standard redox probes and biomolecules revealing the enhanced electro-catalytic activities of FGO and RFGO, and electron transfer rates are simulated theoretically. This study reveals that fluorine not only induces defects in graphitic lattice leading to an enhanced HET process but also can modify the electronic structure of graphene surface.
\end{abstract}

Received 11th May 2014

Accepted 19th June 2014

DOI: $10.1039 / c 4 n r 02563 f$

www.rsc.org/nanoscale and structure are insignificant in electron transfer process, and the electrochemical response will not vary much from single to few layers of graphene. ${ }^{\mathbf{1 0}}$

Doping can modify the electronic structure of graphene, and can also bring interesting physicochemical properties to the graphitic system. ${ }^{\mathbf{1 1 - 1 3}}$ Various functional derivatives of graphene have been synthesized via doping, and fluorinated graphene (FG) is one among them. ${ }^{\mathbf{1 4}, 15}$ Fluorination of graphene will introduce $\mathrm{sp}^{3}$ carbon in $\mathrm{sp}^{2}$ honeycomb matrix, and will alter the electronic structure of graphene. Moreover, highly electronegative fluorine can change the surface properties of graphene with a high thermal stability in to the structure. ${ }^{16}$ Recently, some of these authors studied various multifunctional properties of FG derivatives, and attempted a green synthesis route towards the development of bulk amount of FG. ${ }^{17}$ In this study, we report a phenomenal enhancement in the electron transfer properties of graphene upon fluorine incorporation and demonstrate the fundamental mechanism underlying this enhanced heterogeneous electron transfer (HET) process with the aid of detailed electrochemistry analysis and computational simulation. Detailed HET studies on fluorinated graphene oxide (FGO) (a functional derivative of FG, containing various oxygen functionalities) and reduced fluorinated graphene oxide (RFGO) were conducted for the first time with a negatively charged redox molecule namely $\left[\mathrm{Fe}(\mathrm{CN})_{6}\right]^{3-/ 4-}$.

Extensive studies on the HET processes on glassy carbon and HOPG surfaces have been performed by McCreery et al., However, unlike these systems, the electrochemistry of graphene needs to be understood by giving due consideration to
CSIR-Central Electrochemical Research Institute (CSIR-CECRI), Karaikn
India.E-mail: tn_narayanan@yahoo.com; ssenthilmugam@gmail.com

$\dagger$ Electronic supplementary information (ESI) available. See DOI: $10.1039 / \mathrm{c} 4 \mathrm{nr} 02563 \mathrm{f}$ 
several other factors such as conductivity (way of synthesis), percentage and nature of defects, presence of functionalities, orientation and presence of other elements. ${ }^{18,19}$ Graphene oxide (GO), which is an oxygen rich functional derivative of graphene ${ }^{\mathbf{2 0}}$ is considered as a base material for the bulk synthesis of graphene by various reduction methods. However, graphene produced by this method may contain residual functional groups and defects such as Stone-Wales type, ${ }^{21}$ and these can alter the resultant HET process. ${ }^{22,23}$ The extent of reduction and nature of reduction method carried out also affect the resultant end products; hence, a notable variation in HET rate constant of graphene is observed in different reports. ${ }^{24,25}$ HET studies on similarly synthesized fluorine-doped graphene oxide systems are not available in literature and such studies will fundamentally probe the electron transfer mechanism.

\section{Experimental section}

\section{Synthesis of GO, RGO, FGO and RFGO}

GO has been synthesized using "Improved Synthesis" method. ${ }^{17}$ A 9 : 1 mixture of concentrated $\mathrm{H}_{2} \mathrm{SO}_{4}-\mathrm{H}_{3} \mathrm{PO}_{4}(360: 40 \mathrm{~mL})$ was added to a mixture of graphite flakes $(3.0 \mathrm{~g}, 1 \mathrm{wt}$ equiv., SP-I Bay carbon) and $\mathrm{KMnO}_{4}(18.0 \mathrm{~g}, 6 \mathrm{wt}$ equiv.). The reactants were then heated to $50{ }^{\circ} \mathrm{C}$ and stirred for $12 \mathrm{~h}$. The reaction was cooled to room temperature and poured onto ice with $30 \% \mathrm{H}_{2} \mathrm{O}_{2}$ (3 mL). The material was then washed in succession with $200 \mathrm{~mL}$ of water, $200 \mathrm{~mL}$ of $30 \% \mathrm{HCl}$, and $200 \mathrm{~mL}$ of ethanol (2 times). The material remaining after this multiple-wash process was coagulated with $200 \mathrm{~mL}$ of ether, and the resultant suspension was filtered over a PTFE membrane with a $0.22 \mu \mathrm{m}$ pore size. Fluorinated GO (FGO) has also been prepared using a similar method taking fluorinated graphite polymer (Alfa Aesar 42537) as the starting material and scooping the phase separated top part, as has been reported in previous work. ${ }^{17}$ Both GO and FGO were subjected to a controlled thermal reduction at 90 ${ }^{\circ} \mathrm{C}$ under hydrazine hydrate atmosphere for 12 hours in vacuum oven to obtain graphene (RGO) and FG (RFGO).

STEM measurements were carried out using JEOL 2100 fieldemission gun transmission electron microscope, and micro Raman analysis was carried out using Renishaw Raman Microscope. Raman analysis was carried out on the powder samples with $633 \mathrm{~nm}$ laser excitation. Laser intensity was kept $\sim 1 \%$ and the exposure time was 30 seconds. A $50 \times$ lens was used to focus the laser beam. Bruker FT-Infrared instrument used to conduct FT-IR measurements. Bruker FT Nuclear Magnetic Resonance (NMR) spectrometer (400 MHz) was used for ${ }^{19} \mathrm{~F}$ NMR spectrum acquiring.

Fabrication of graphene electrodes. $2.5 \mathrm{mg}$ of synthesized graphene material was dispersed in $500 \mu \mathrm{l}$ millipore water and $5 \mu \mathrm{l}$ of this dispersed material was drop-casted on well-polished glassy carbon (GCE) surface for the modification. Then, electrodes were allowed $30 \mathrm{~min}$ for drying. These modified electrodes were used for all the electrochemical experiments. The geometrical area of GCE (BASi Analytical Instruments) is $0.07 \mathrm{~cm}^{2}$.

Electrochemical studies. CHI instrument Inc (model no. 900B) was used for all potentiodynamic experiments.
Heterogeneous electron transfer studies were performed on different types of GO modified electrodes using cyclic voltammetry in aqueous electrolyte $1 \mathrm{M} \mathrm{KCl}$ containing $1 \mathrm{mM}$ $\mathrm{K}_{3}\left[\mathrm{Fe}(\mathrm{CN})_{6}\right]$ solutions at the scan rate of $0.1 \mathrm{~V} \mathrm{~s}^{-1}$ from the switching potential range between $0.2 \mathrm{~V}$ and $0.6 \mathrm{~V}$. BAS-IM6 was used to obtain the Nyquist plot of the electrochemical impedance spectroscopy at formal potential in aqueous electrolyte $1 \mathrm{M}$ $\mathrm{KCl}$ containing $1 \mathrm{mM} \mathrm{K}_{3}\left[\mathrm{Fe}(\mathrm{CN})_{6}\right]$ solutions with a frequency range from $100 \mathrm{kHz}$ to $0.05 \mathrm{~Hz}$. $\mathrm{Ag} / \mathrm{AgCl}$ reference electrode was used for entire electrochemical studies. A three electrode cell set up was used for cyclic voltammetry.

Computational simulations studies. Digielch electrochemical simulation software (Digielch 7 professional V7, Model no. 987-00076) was used for the simulation of electrochemical experimental results.

\section{Results and discussion}

In the present investigation, 4-different systems, namely, fluorinated graphene oxide (FGO), GO, reduced fluorinated graphene oxide (RFGO) and graphene (reduced graphene oxide, RGO), were subjected to HET studies. Detailed structure and synthesis procedures of each of these materials have been discussed in our previous reports and briefly explained in the experimental section. In a nutshell, all these materials were synthesized via the same chemical route and reduction of oxide systems have been conducted in a similar way to ensure the same extent of reduction of oxygen functionalities. Similar to our earlier reports and those of many others, all these materials are layered 2-dimensional (2D) materials containing 1-2 atomic layer thickness, similar BET surface area (BET surface area of powder samples $\sim 140$ to $150 \mathrm{~m}^{2} \mathrm{~g}^{-1}$ ) and lateral width. High angle annular dark field image of FGO collected using a scanning transmission electron microscope is shown in Fig. 1A. Atomic layering is evident from this image. This FGO contains $\sim 23 \%$ of $\mathrm{F}$ by atomic percentage $(64.61 \%$ carbon and $11.45 \%$ oxygen). Thermal treatment of FGO with hydrazine vapours results in the reduction of its oxygen functionalities, giving rise to a reduced form known as RFGO (details of reduction procedure are given in the experimental section, and the extent of reduction is discussed in the next section).

FT-IR spectra of all these samples were shown in Fig. 1B. All the features of the FT-IR spectra of these samples are reported in our previous reports. In particular, the characteristic difference between fluorinated and non-fluorinated samples is the presence of $\mathrm{C}-\mathrm{F}$ bond in fluorinated samples at $1204 \mathrm{~cm}^{-1}$. Moreover, the similar reduction of GO and FGO leads to the same extent of removal of oxygen functional groups, as it is clear from the FTIR spectra of reduced samples (RGO and RFGO). In the cases of RGO and RFGO also the major difference in the amount of functional groups is only in $\mathrm{C}-\mathrm{F}$. This indicates that except the presence of fluorine, the chemical structure of both RGO and RFGO are similar. ${ }^{19} \mathrm{~F}$ NMR spectrum of FGO is recorded after dispersing FGO powder in chloroform, and the details are shown in supporting information. The NMR spectrum shows two distinct peaks placed near $\sim-180 \mathrm{ppm}$, indicating the presence of covalent $\mathrm{C}-\mathrm{F}$ bonds. Furthermore, ${ }^{19} \mathrm{~F}$ 

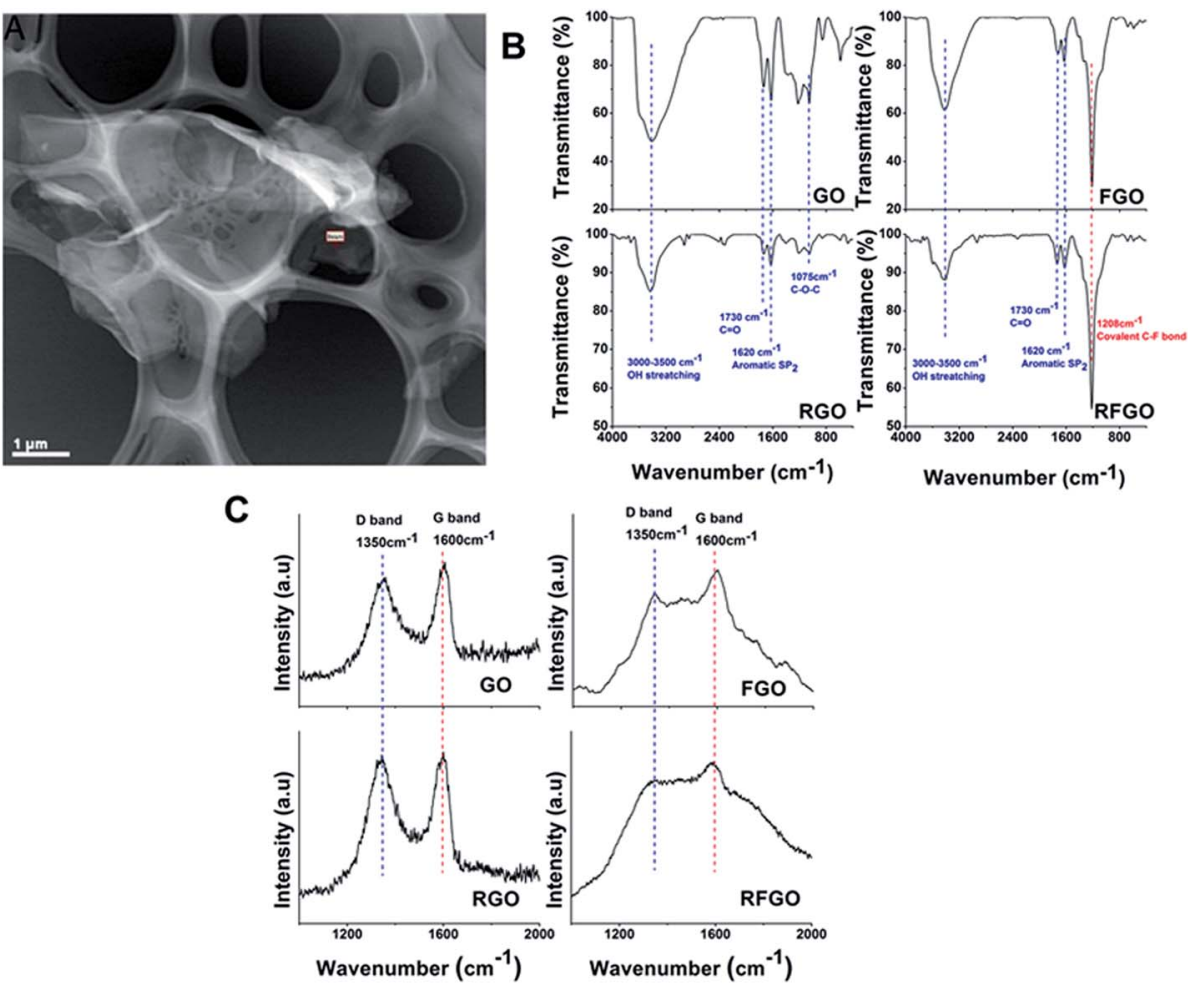

Wavenumber $\left(\mathrm{cm}^{-1}\right)$

Wavenumber $\left(\mathrm{cm}^{-1}\right)$

D band $G$ band

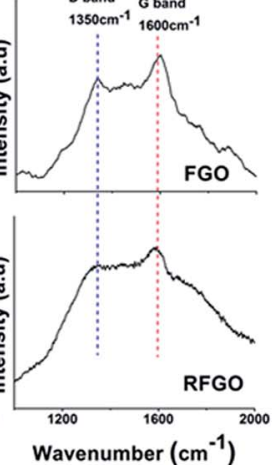

Fig. 1 (A) High angle annular darkfield image of FGO, showing the atomically thin layered structure of FGO. (B) FTIR spectra of different graphene oxides, (C) Raman spectra of different graphene oxides.

NMR also shows the presence of tertiary alkyl fluorides and confirms the presence of more fluorine in the basal plane than at the edges.

For further probing the nature of defects present in these systems, a detailed micro-Raman analysis is performed and the results are appended in the Fig. 1C. It is clear from the Raman analysis and from our earlier studies that the samples show clear signatures of graphitization in RFGO and RGO. A relatively high $\mathrm{sp}^{3}$ content in RFGO is due to the presence of a large amount of fluorine.

Electrochemistry of GO/RGO and FGO/RFGO were studied using cyclic voltammetry (details of the experiment are given in the Experimental section) and electrochemical impedance spectroscopy. Fig. 2A-D shows the comparison of cyclic voltammogram (CV) of graphene oxide-based electrodes (electrode synthesis is explained in the Experimental section) in mixture of $1 \mathrm{mM} \mathrm{K}_{3}\left[\mathrm{Fe}(\mathrm{CN})_{6}\right]$ and $1 \mathrm{M} \mathrm{KCl}$ solutions at a scan rate of $0.1 \mathrm{~V}$ $\mathrm{s}^{-1}$. A quasi-reversible, negatively charged redox molecule, i.e., potassium ferricyanide, is used for HET studies. GO shows negligible current for the HET of $\left[\mathrm{Fe}(\mathrm{CN})_{6}\right]^{3-/ 4-}$ and its corresponding redox peak potential difference is around $0.2 \mathrm{~V}$ (Fig. 2A). This indicates that GO is blocking the HET electron transfer due to electrostatic repulsion between negatively charged ferricyanide species, ${ }^{24}$ and behaves like an insulating material due to the presence of large varieties of oxygen containing functional groups such as $-\mathrm{C}=\mathrm{O},-\mathrm{COOH},-\mathrm{CHO}$, and $-\mathrm{OH}$. It has been established that oxygen functional groups present in GO may lead to the electrostatic repulsion resulting to a larger electron tunnelling distance and more sluggish kinetics. ${ }^{24}$

A similar study has been conducted on FGO (Fig. 2B). Remarkably, FGO shows a well-defined peak at $0.220 \mathrm{~V}$, corresponding to the HET of $\left[\mathrm{Fe}(\mathrm{CN})_{6}\right]^{3-/ 4-}$ with redox peak potential difference $0.11 \mathrm{~V}$. It is assumed that FGO, having a lot of oxygen containing functional groups with covalent $\mathrm{C}-\mathrm{F}$ bonds, is expected to retard the HET and will act like GO in the HET process. However, contrary to this expectation, FGO behaves like a very good electrode material compared to GO. This observation leads to the conclusion that fluorine plays a seminal role in distinguishing the HET processes in these two materials, which are otherwise similar in terms of chemical (except the presence of fluorine in $\mathrm{FGO}$ ) and physical structure.

Next, we have compared the cyclic voltammograms (CV) of RGO and RFGO with GO and FGO. These reduced forms of graphene oxides show a higher peak current and low redox peak potential difference (Fig. 2C and D) than their corresponding. unreduced forms of graphene oxides in HET processes. This indicates that the functional groups are substantially removed (as seen in FT-IR spectra) after the hydrazine reduction; thus, they play a key role in hindering the electron transfer process, correlating well with the other reports. ${ }^{24}$ Though there are some contradictory arguments on the role of functional groups on the carbon process in HET, this enhanced electron transfer in the case of reduced systems may be due to the reduced negative charge and enhanced electronic conductivity. ${ }^{26,20}$ However, no study has been conducted so far to correlate the conductivity of 

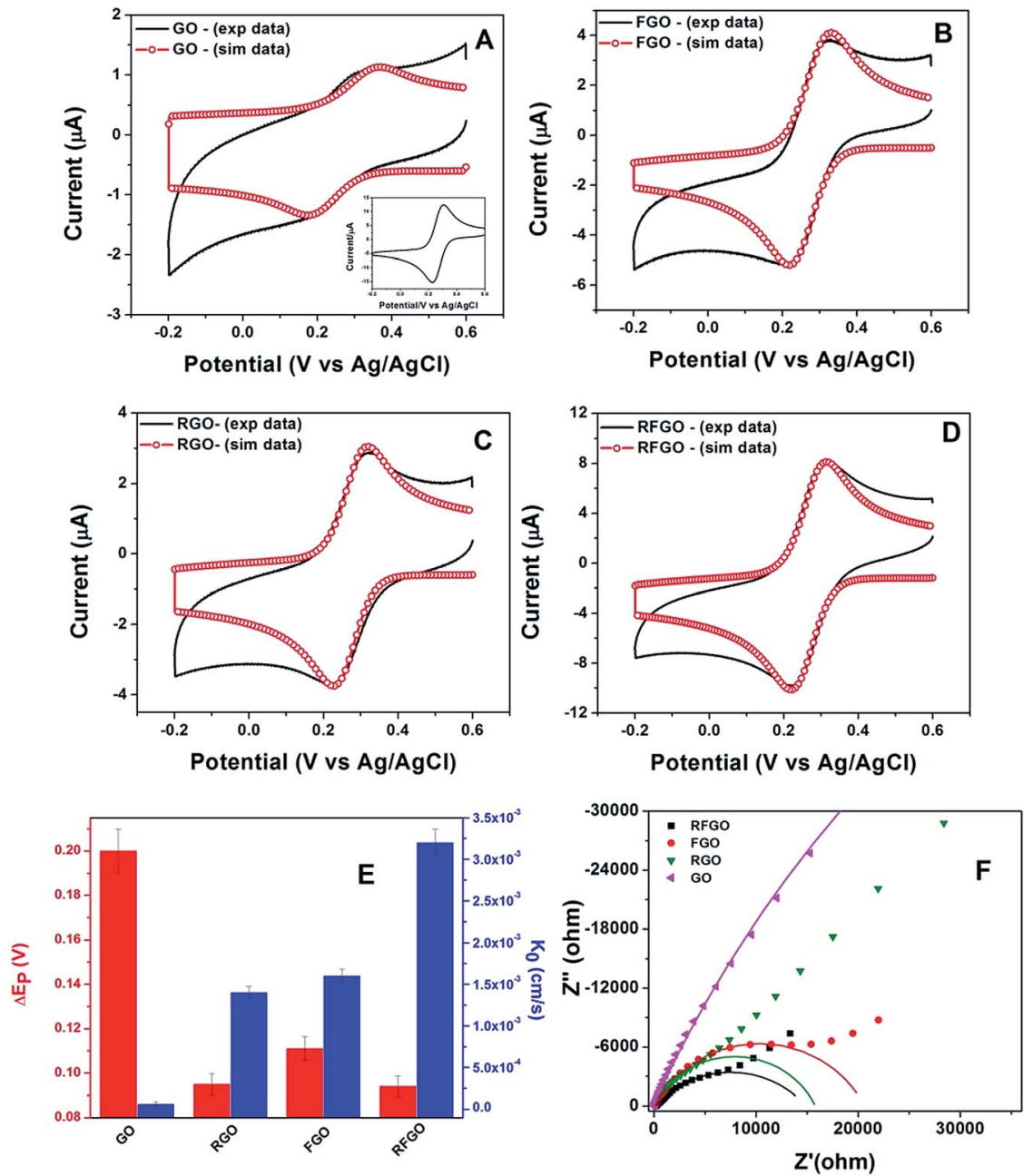

Fig. 2 Cyclic voltammograms obtained using different graphene oxide material ((A) GO, (B) FGO, (C) RGO, (D) RFGO) modified on glassy carbon electrode at scan rate of $0.1 \mathrm{~V} \mathrm{~s}^{-1}$ in aqueous electrolyte, i.e., $1 \mathrm{M} \mathrm{KCl}$ containing $1 \mathrm{mM} \mathrm{K}{ }_{3}\left[\mathrm{Fe}(\mathrm{CN})_{6}\right]$ solutions (black line-experimental data, red colour circle-simulation data). (A) Inset: bare GCE cyclic voltammogram obtained in the abovementioned conditions. (E) Plot of anodic and cathodic peak potential difference of $\left[\mathrm{Fe}(\mathrm{CN})_{6}\right]^{4-13-}$ and rate constant values of different $\mathrm{GO}$ surfaces. (F) Nyquist plot obtained using EIS measurements of different $\mathrm{GO}$ surfaces in aqueous electrolyte, i.e., $1 \mathrm{M} \mathrm{KCl}$ containing $1 \mathrm{mM} \mathrm{K} \mathrm{K}_{3}\left[\mathrm{Fe}(\mathrm{CN})_{6}\right]$ solutions from $100 \mathrm{kHz}$ to $0.05 \mathrm{~Hz}$. Dotted curve is experimental data; solid line corresponds to fitted semi-circle curve.

modified electrode materials, and their heterogeneous electron transfer. ${ }^{27}$

Moreover, the HET process was simulated using Digielch 7 professional V7 software. Here, experimental parameters were used to simulate the $\mathrm{CV}$ for the four samples (GO, FGO, RGO and RFGO) and fitted with the faradaic portion of the experimental data. The HET rate constants $\left(K^{\circ}\right)$ were determined through digital simulation of cyclic voltammetric $I-V$ curve (details of experimental parameters are given in the Experimental section). Comparing the HET rate constants, it is observed that HET rates (in $\mathrm{cm} \mathrm{s}^{-1}$ ) follow the following order: $\operatorname{RFGO}\left(3.2 \times 10^{-3}\right)>\mathrm{FGO}\left(1.6 \times 10^{-3}\right)>\operatorname{RGO}\left(1.4 \times 10^{-3}\right)>\mathrm{GO}$ $\left(6.2 \times 10^{-5}\right)$. For clear understanding, the averaged redox peak potential difference and obtained HET rate constant of $\left[\mathrm{Fe}(\mathrm{CN})_{6}\right]^{4-/ 3-}$ at various forms of graphene oxide shown in Fig. 2E. This indicates that fluorine is playing a significant role in HET processes, apart from the effects of the oxygen functional group effects, slowing down the electron transfer kinetics.

Furthermore, we probed the surface chemistry of these GO materials using electrochemical impedance spectroscopy (EIS) to evaluate HET resistance between the electrode material and $\left[\mathrm{Fe}(\mathrm{CN})_{6}\right]^{3-14-}$. EIS performed at the formal potential of $\left[\mathrm{Fe}(\mathrm{CN})_{6}\right]^{3-14-}$ to measure the charge transfer resistance of different samples (Fig. 2F). GO exhibits a higher charge transfer resistance of around $233 \mathrm{k} \Omega$ due to its high amount of oxygen functionalities with large number of $\mathrm{sp}^{3}$ domains. FGO shows less charge transfer resistance $(\sim 20.53 \mathrm{k} \Omega)$ than GO, even with the presence of oxygen containing functional groups (similar amount, FT-IR) on its surface. EIS data is consistent with the cyclic voltammetry results. Fluorine-doped systems show high amount of defects density so these may be responsible for enhanced HET process. This is in agreement with a very recent report by Pumera et al., in which they showed the enhanced HET kinetics with the amount of fluorine in fluorinated 
graphite polymers. ${ }^{28}$ In the case of RGO $(15.8 \mathrm{k} \Omega)$ and RFGO $\left(14.6 \mathrm{k} \Omega\right.$ ), lower $R_{\mathrm{ct}}$ was observed than those corresponding unreduced forms of graphene oxides. This HET resistance reflects in low redox peak potential difference $\left(\Delta E_{\mathrm{p}}\right)$ of molecular probe $\left[\mathrm{Fe}(\mathrm{CN})_{6}\right]^{3-/ 4-}$ cyclic voltammetry. The chemical reduction of GO/FGO will introduce additional defects on honeycomb lattice in the form of dangling bonds, Stone-Wales defects and formation of large number of small $\mathrm{sp}^{2}$ domains. ${ }^{29}$ These additional defects may be responsible for enhanced electron transfer kinetics in reduced systems. In addition, the reduction of oxygen functionalities will also improve the electronic conductivity of the materials.

Hence, in the case of fluorinated graphene derivatives, the presence of a large number of defect sites arising due to the presence of fluorine and other oxygen functionalities (as can be seen in Raman data) is responsible for the augmented HET process. Furthermore, the presence of fluorine in the graphitic matrix is reduces the surface energy and hence make the surface more hydrophobic. ${ }^{17}$ Hence, even oxygenated FGO has been reported for its amphiphobic properties by some of the authors. Hence, the negatively charged redox molecule will not get repelled by the electrode, although it contains negatively charged oxygen functionalities. ${ }^{30}$ This is also one of the potential possibilities for the improved heterogeneous electron transfer properties of FGO. Recently, Pumera et al. reported the HET kinetics of halogenated graphene oxides, in which the electron transfer rate increases with the decrease in atomic number of the doped halogen (transfer rates track the following order, Cl-TRGO > Br-TRGO > I-TRGO) (where TRGO means thermally reduce graphene oxide). ${ }^{31}$ In the present work, the HET rates obtained for RFGO is $3.2 \times 10^{-3} \mathrm{~cm} \mathrm{~s}^{-1}$. This value is higher than that reported for Cl-TRGO $\left(2.52 \times 10^{-3} \mathrm{~cm} \mathrm{~s}^{-1}\right)$. Hence, this also proves the role of the atomic weight of the doped element in graphene oxide materials for determining the resultant HET.

The electrooxidation of biologically important molecules such as ascorbic acid (AA), dopamine (DA), and uric acid (UA) using graphene electrodes are always dependent on the alignment of graphene electrodes and the nature of functional groups present on graphene surfaces. ${ }^{11}$ By studying the electrocatalytic behavior of these kinds of molecules on FGO, one can understand the intrinsic properties of the FGO surface. Fig. 3A-C shows the CVs of $1.0 \mathrm{mM}$ AA, DA, and UA oxidation in PBS $(\mathrm{pH}=7.4)$ at different graphene oxide materials modified glass carbon electrode. AA oxidation is a chemically irreversible process, and the electrode kinetics depend on oxidation potential $\left(E_{\text {pox }}\right)$. It can be seen from Fig. $3 A$ that the AA oxidation is fully hindering with GO surface, which can be due to the electrostatic repulsion between rich oxygenated functional groups in GO and negatively charged AA molecule. Unlike GO, RGO shows AA oxidations and anodic peak current start at $0.2 \mathrm{~V}$, indicating hydrazine reduction leading to the effective removal of the oxygenated functional groups. Interestingly, the FGOmodified GC electrode shows significantly lower overpotential with anodic peak current starting at $0.0 \mathrm{~V}$, indicating fast electron transfer kinetics $(0.2 \mathrm{~V}$ less anodic shifts in oxidation potential than RGO) even with the presence of more oxygen
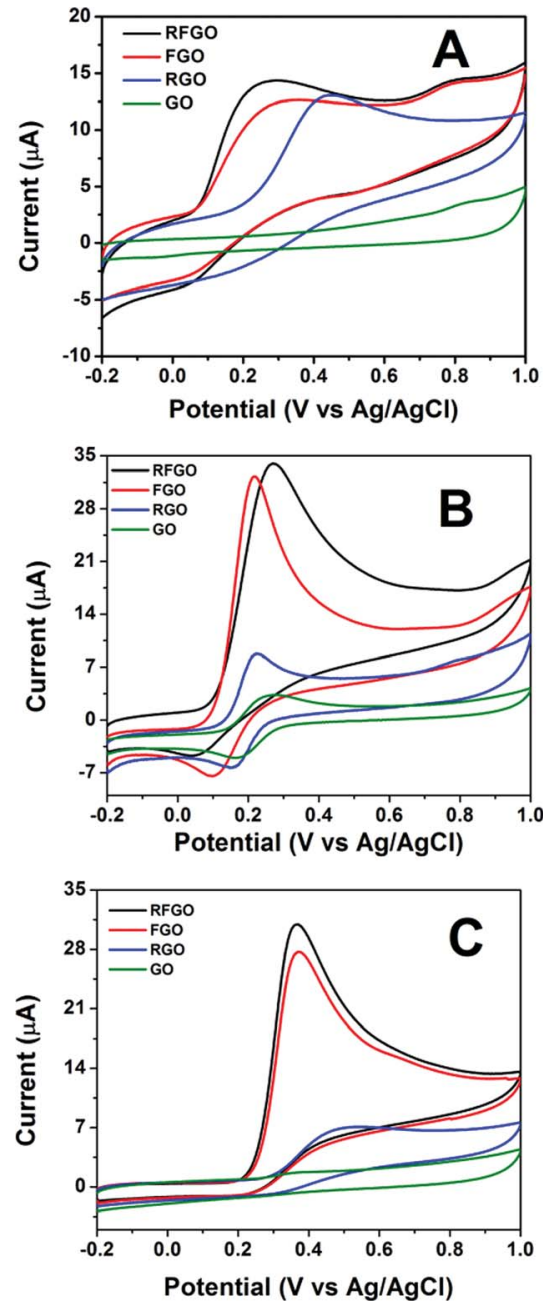

Fig. 3 Cyclic voltammograms obtained in functionalized $\mathrm{GO}$ surfaces in PBS (pH 7.4) containing (A) $1 \mathrm{mM}$ ascorbic acid (B) $1 \mathrm{mM}$ dopamine (C) $1 \mathrm{mM}$ uric acid solutions at scan rate of $0.1 \mathrm{~V} \mathrm{~s}^{-1}$.

containing functional groups than that in RGO. In the case of RFGO, AA oxidation peak starts at the same potential of $0.0 \mathrm{~V}$ and only small peak current $(2 \mu \mathrm{A})$ enhancement is observed compared to FGO surface. This indicates that there was no significant influence of the oxygen functional present on the FGO surface. As reported in previous literature, AA oxidation is a surface sensitive electrochemical reaction, ${ }^{2}$ i.e., sensitive to surface chemistry and microstructure, as well as density of electronic states near the formal potential. ${ }^{1,32}$ Unlike AA, DA oxidation peak is noticeable at even GO surface (Fig. 3B). This is because of electrostatic attraction between negatively charged GO surface and positively charged DA molecules in PBS ( $\mathrm{pH}=$ 7.4) condition. In comparison to GO electrode, RGO shows a lower DA oxidation potential $(0.1 \mathrm{~V})$ and a higher peak current $(10 \mu \mathrm{A})$. In the case of FGO, superior catalytic activity was observed for DA oxidation, in term of less anodic over potential $(0.05 \mathrm{~V})$ with 3.3 times $(33 \mu \mathrm{A})$ higher current than GO surface. Like AA oxidation, DA oxidation at RFGO surface was also not much affected in terms of oxidation potential and peak current compared to FGO. However, it is established that DA is less 
sensitive to the surface oxygen functional groups on graphene surface; ${ }^{19}$ hence, this study reveals that fluorine doping on graphene oxide may have a prominent role in altering the density of electronic states near Fermi level.

Finally, we studied UA oxidation on different GO surfaces, as shown in Fig. 3C. UA is also negatively charged in PBS $(\mathrm{pH}=$ 7.4) condition. Moreover, it also repels like the AA molecule on GO surface and no characteristic oxidation behaviour is observed on GO. However, in the case of FGO surface, UA oxidation peak starts at $0.208 \mathrm{~V}(0.090 \mathrm{~V}$ less anodic shifts in oxidation potential than RGO) with 4.5 times higher current than RGO. This also further confirms that the surface of FGO has higher catalytic nature than GO and RGO. The enhancement of fast electron transfer kinetics of AA, DA and UA on RFGO and FGO is probably due to the abundant microstructure, defects and density of electronic states near the formal potential. Some theoretical work is needed for understanding the electronic states of fluorine-doped graphene oxide and these studies are in progress. Moreover, the electrochemical studies on fluorinated graphene derivatives indicate that fluorine not only changes the physicochemical properties of the graphene surface but may also alter its electronic and magnetic properties, in agreement with our earlier studies on similar fluorinated systems. $^{33}$

\section{Conclusions}

A detailed electrochemical study has been conducted on various functional derivatives of doped and undoped graphene oxide surfaces to prove the role of defects in determining the net electron transfer kinetics. It is observed that the presence of fluorine in graphene oxide surface induces more defects on graphitic surface, leading to an enhanced HET kinetics than the undoped counter parts. Moreover, this study also reveals that fluorine can alter the electronic states of graphene surface. The observed less anodic over potential and higher peaks currents for electrooxidation of biologically important molecules of AA, DA and UA with FGO/RFGO surfaces indicate the efficient electro catalytic nature of fluorinated materials than GO and RGO electrodes. This study opens the possibilities for developing various point-of-care devices using fluorinated graphene derivatives, and also opens a new avenue for tuning the electron transfer properties of electrodes by changing the nature of dopants.

\section{Acknowledgements}

Among the authors, SB thanks CSIR for the SRF fellowship. TNN acknowledges the financial support from CSIR-CECRI in the form of institution start-up fund (OLP 0087) and also acknowledges DST for financial support in the form of DSTFAST Track scheme (SB/FTP/PS-084/2013: GAP26/13). SS acknowledges CSIR-Multifun (CSC0101). TNN also acknowledges Prof. P. M. Ajayan, Rice University, for his support.

\section{Notes and references}

1 R. L. McCreery, Chem. Rev., 2008, 108, 2646.

2 P. Chen and R. L. McCreery, Anal. Chem., 1996, 68, 3958.

3 M. Pumera, Chem. Soc. Rev., 2010, 39, 4146.

4 K. R. Ratinac, W. Yang, J. J. Gooding, P. Thordarson and F. Braet, Electroanalysis, 2011, 23, 803.

5 T. A. Silva, H. Zanin, E. Saito, R. A. Medeiros, F. C. Vicentini, E. J. Corat and O. Fatibello-Filho, Electrochim. Acta, 2014, 119, 114.

6 S. V. Morozov, K. S. Novoselov, M. I. Katsnelson, F. Schedin, D. C. Elias, J. A. Jaszczak and A. K. Geim, Phys. Rev. Lett., 2008, 100, 16602.

7 S. Park and R. S. Ruoff, Nat. Nanotechnol., 2009, 4, 217.

8 S. Alwarappan, A. Erdem, C. Liu and C.-Z. Li, J. Phys. Chem. C, 2009, 113, 8853.

9 D. a. C. Brownson, L. J. Munro, D. K. Kampouris and C. E. Banks, RSC Adv., 2011, 1, 978.

10 M. S. Goh; M. Pumera. Anal. Chem. 2010, 82, 8367.

11 Z.-H. Sheng, X.-Q. Zheng, J.-Y. Xu, W.-J. Bao, F.-B. Wang and X.-H. Xia, Biosens. Bioelectron., 2012, 34, 125.

12 F. Karlický, K. Kumara Ramanatha Datta, M. Otyepka and R. Zbořil, ACS Nano, 2013, 7, 6434.

13 V. Georgakilas, M. Otyepka, A. B. Bourlinos, V. Chandra, N. Kim, K. C. Kemp, P. Hobza, R. Zboril and K. S. Kim, Chem. Rev., 2012, 112, 6156.

14 P. Gong, Z. Wang, Z. Li, Y. Mi, J. Sun, L. Niu, H. Wang, J. Wang and S. Yang, RSC Adv., 2013, 3, 6327.

15 J. T. Robinson, J. S. Burgess, C. E. Junkermeier, S. C. Badescu, T. L. Reinecke, F. K. Perkins, M. K. Zalalutdniov, J. W. Baldwin, J. C. Culbertson, P. E. Sheehan and E. S. Snow, Nano Lett., 2010, 10, 3001.

16 P. Chantharasupawong, R. Philip, N. T. Narayanan, P. M. Sudeep, A. Mathkar, P. M. Ajayan and J. Thomas, J. Phys. Chem. C, 2012, 116, 25955.

17 A. Mathkar, T. N. Narayanan, L. B. Alemany, P. Cox, P. Nguyen, G. Gao, P. Chang, R. Romero-Aburto, S. a. Mani and P. M. Ajayan, Part. Part. Syst. Charact., 2013, 30, 266.

18 M. G. Sheets; W. Li; C. Tan; M. A. Lowe. 2011, 2264.

19 D. a C. Brownson, M. Gómez-Mingot and C. E. Banks, Phys. Chem. Chem. Phys., 2011, 13, 20284.

20 C. X. Lim, H. Y. Hoh, P. K. Ang and K. P. Loh, Anal. Chem., 2010, 82, 7387.

21 C. Gómez-Navarro, J. C. Meyer, R. S. Sundaram, A. Chuvilin, S. Kurasch, M. Burghard, K. Kern and U. Kaiser, Nano Lett., 2010, 10, 1144.

22 Y. Shao, J. Wang, H. Wu, J. Liu, I. A. Aksay and Y. Lin, Electroanalysis, 2010, 22, 1027.

23 D. a C. Brownson, D. K. Kampouris and C. E. Banks, Chem. Soc. Rev., 2012, 41, 6944.

24 A. Ambrosi, C. K. Chua, A. Bonanni and M. Pumera, Chem. Mater., 2012, 24, 2292.

25 A. Ambrosi, A. Bonanni, Z. Sofer, J. S. Cross and M. Pumera, Chemistry, 2011, 17, 10763.

26 X. Ji, C. E. Banks, A. Crossley and R. G. Compton, ChemPhysChem, 2006, 7, 1337. 
27 M. Pumera, Chem. Rec., 2009, 9, 211.

28 X. Chia, A. Ambrosi, M. Otyepka, R. Zbořil and M. Pumera, Chemistry, 2014, 20, 1.

29 M. Acik and Y. J. Chabal, Jpn. J. Appl. Phys., 2011, 50, 070101.

30 M. R. Deakin, P. M. Kovach, K. J. Stutts and R. M. Wightman, Anal. Chem., 1986, 58, 1474.

31 H. L. Poh, P. Šimek, Z. Sofer and M. Pumera, Chemistry, 2013, 19, 2655.
32 L. Tang, Y. Wang, Y. Li, H. Feng, J. Lu and J. Li, Adv. Funct. Mater., 2009, 19, 2782.

33 R. Romero-Aburto, T. N. Narayanan, Y. Nagaoka, T. Hasumura, T. M. Mitcham, T. Fukuda, P. J. Cox, R. R. Bouchard, T. Maekawa, D. S. Kumar, S. V. Torti, S. a. Mani and P. M. Ajayan, Adv. Mater., 2013, 25, 5632. 